\title{
Research on Optimization Control Technology for Coordinated of Source-grid-load Based on Balance of Power Supply and Demand
}

\author{
Jingkai Zhu ${ }^{1, a}$, Xiaotian Wang ${ }^{2, b}$ and Xiaofeng $\mathrm{Xu}^{3, \mathrm{c}}$ \\ ${ }^{1}$ State Grid Shanghai Municipal Electric Power Company, China \\ ${ }^{2}$ State Grid Shanghai Municipal Electric Power Company, China \\ ${ }^{3}$ North China Electric Power University, China \\ a1163600676@qq.com , bxtwang1982@163.com , ‘xxfncepu@163.com
}

\begin{abstract}
Keywords: Power supply and demand balance, Source-grid-load, Coordination and optimization, Electric power system

Abstract. The construction and development of UHV power grids has changed the energy allocation mode which was excessively relied on coal transportation and power development mode which balanced locally in the past. However, with the development of UHV technology, the security contradiction of the large power grid has become increasingly prominent. This article studies how to carry out coordinated optimization control of the source-grid-load on the basis of maintaining the balance of power supply and demand. Firstly, the basic model analysis of optimization control for source-grid-load coordination is carried out. Based on this, the need for coordinated optimization of source-grid-load is analyzed, and then the control technology of source-grid-load coordination and optimization operation is proposed. The purpose is to solve the safety problems for the operation of large power grids in the context of UHV technology and further improve the operational stability of the power grid.
\end{abstract}

\section{Introduction}

The improvement of China's energy supply capability is restricted by two constraints: uneven distribution of energy resources and uneven economic development ${ }^{[1]}$. In recent years, China's energy development has accelerated to the West and the north, and the distance between the energy base and the load center is getting farther and farther away. The construction and development of UHV power grid has greatly improved our country's ability to optimize the allocation of resources. A series of problems, such as coal and electricity capacity, repeated rise in coal and electricity price and environmental pollution, have been solved ${ }^{[2]}$.

With the rapid development of UHV power grids and the increasing transmission power, the scale of load in the eastern and central load centers is increasing. One is that the conventional power supply is replaced by a large scale, the equivalent rotation inertia of the terminal power grid continues to decline, and the power supply safety regulation ability of the large terminal power grid is seriously inadequate. Two is the single DC power supply scale continuously increasing. Once lost, the terminal power grid is transient in large amount of power loss, the frequency of the power grid decreases rapidly, and a large number of transect transect. The limit and standby capacity are seriously inadequate and face a large area blackout risk. Three is an UHV direct current transmission corridor centralized, dense DC drop range, complex external environment along the line, multiple lines crossing points, caused by natural disasters and serious fault at the end of the power grid, the possibility of a large number of UHV transmission lines at the same time, once the possibility of failure, once occurred There is a risk of collapse in the grid ${ }^{[3]}$.

The coordination and optimization between power grid and power supply and load three can guarantee the transient, short-time and time balance of power supply in the emergency state, so as to ensure the stable operation of the power grid . However, the transformation from "power regulation" to "power regulation" and "load regulation" needs a certain control technology as support ${ }^{[5]}$. 
Therefore, it is necessary to study the coordinated optimization control technology of Source-grid-load.

\section{Basic model of coordinated optimization control of Source-grid-load}

The coordinated optimal control of Source-grid-load is essentially a solution to the complex power dynamic balance problem of power system. From this point of view, the connotation of interaction is discussed from the perspective of "disturbance response", and a coordinated optimal control model of Source-grid-load is established. The so-called disturbance refers to the various internal or external factors that cause undesired changes in the amount of control, which can be divided into source disturbance, network disturbance and charge disturbance from the subject of disturbance. Response is a specific form of interaction. For different types of disturbances, the source, network, and charge will respond accordingly, forming an interactive behavior. Generally speaking, in the analysis of the interaction mode of the power grid, the disturbance and response of the source and the load side directly affect the power balance of the power grid, and the disturbance and response of the network mainly affect the operation state of the power grid.

Based on the "disturbance response" model, the following 6 main interactive forms can be summarized, as shown in Table 1.

Table 1. Basic model of coordinated optimization control of Source-grid-load

\begin{tabular}{|l|l|l|l|}
\hline Disturbance & Response & Interactive mode & Typical form of expression \\
\hline load & Source & Source and load action & Traditional interactive model \\
\hline Source & load & Load and Source action & Load response power supply \\
\hline grid & grid & Grid and grid action & UPFC Flexible control technology \\
\hline grid & load & Grid and load action & Load shift and demand response \\
\hline grid & Source & Grid and source action & Primary frequency modulation \\
\hline Source & Source & Source and source action & wind-solar-storage hybrid power system \\
\hline
\end{tabular}

\section{Demand analysis of coordinated optimal control of Source-grid-load}

In the process of operation, the power grid must meet the balance of supply and demand in essence, use the mass of interactive resources in the power system, adjust the demand of different scenes flexibly, and change from power regulation to load regulation and power regulation, so as to realize the balance of supply and demand of power grid and power and load. For the supply and demand balance of different time requirements, when the power grid frequency fluctuates, the millisecond level / second level excises the interruptible load, realizes the instantaneous / short-time balance of the power supply and demand, uses the economic incentive means to guide the users to arrange the production reasonably, and optimizes the load of the power supply, and realizes the balance of the power supply and demand period. In the case of emergency, the power grid scheduling is in a rigid scene, with the priority of security and reliability, and a series of interactive behavior should meet the interaction performance requirements within a certain time range and the range of control capacity, so that the power grid can reduce the loss in time from the emergency. Under normal circumstances, the power grid scheduling is in a flexible scene, taking the priority of economy and efficiency as the goal, and arranging all the schedulable resources in the area in order to achieve economic and efficient optimization. The theoretical block diagram of large scale source network friendly interaction target needs is shown in Fig.1. 


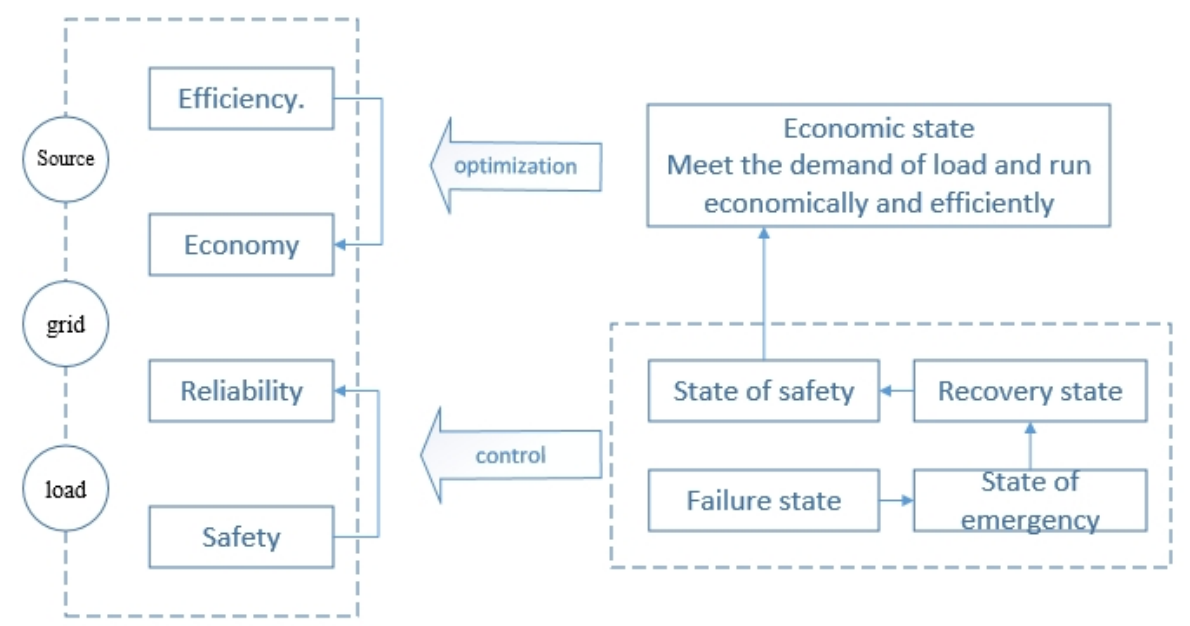

Fig.1. Theoretical block diagram of target demand for friendly interaction between Source-grid-load

Transient and short-time demand for power balance. Instantaneous balance means to provide fast control means for the power shortage fault of power grid. It can remove the interruptible load in millisecond level, realize the instantaneous balance of power supply and demand, and provide the professional means and key resources for the government to deal with the large area blackout. Short time balance means that in the case of emergency disposal of power grid fault and insufficient power resource adequacy, second level excision can interrupt the load, achieve short balance of supply and demand, and give play to the equivalent power of power supply with interruptible load.

For the large receiving power grid characterized by UHV AC and DC mixing, the equivalent inertia and stability regulation ability of the generator in the area is greatly reduced, and the AC and DC power grid is prone to cascading failures. Once the DC closed or commutation failure faults occur, the large power vacancy outside the region will be caused, and the stability of frequency, power angle and voltage will be solved. It follows. The safe operation of power grid is facing great difficulties, and the following four problems need to be solved.

I Guard against the rapid fall of the frequency at the beginning of the failure, and take emergency load control measures in $1 \mathrm{~s}$.

I In order to solve the limit of the main channel power flow, the $15 \mathrm{~min}$ control is in place, and the second level control load can be taken.

I The inter provincial connection line power overuse can be solved. After the failure, the interprovincial contact line of the provincial power grid may be overused, and the $30 \mathrm{~min}$ control is in place, and the minute load control measures can be taken if the system is not enough.

I To solve the shortage of power grid rotation reserve, because of the loss of DC power, the power grid rotation reserve is insufficient and the $1 \mathrm{H}$ control is in place, and the minute load control measures can be taken.

Demand for power balance in time period. Time balance refers to the comprehensive use of economic incentives to guide users to arrange production reasonably, to optimize the shift of electricity load, to realize the balance of supply and demand, and to improve the utilization efficiency of power generation resources.

The demand for electrical load needs to be met at any time. This is a "rigid" feature, and some loads can be adjusted in a certain range according to the needs. We call it a flexible load. The flexible load includes both the industrial load, the commercial load and the traditional load of the air conditioning and refrigerator in the living load of the power users, as well as the bidirectional controllable load such as storage energy and electric vehicle. These demand side response resources can respond to the demand of the power grid in real time and participate in the balance of power supply and demand. Using these demand side response resources to balance the fluctuation of intermittent energy and improve the acceptance of intermittent energy, and reduce the peak valley difference by shifting the peak to the valley, it is of great significance to the efficient operation of the system. 


\section{Key technology of coordinated optimization control of Source-grid-load}

Coordinated control technology of source network with millisecond. The UHV fault control mode is divided into two types: automatic control and auxiliary decision control. Automatic control is divided into emergency and emergency control mode. In the case of UHV, the emergency control mode receives the command of the load capacity of the co control station, combines the local frequency anti error criterion, and realizes the rapid removal of the interruptible load of the pre graded grouping by the safety and stability control system. The sub emergency control mode is aimed at the problem of overloading of the important section in the near area of the ultra high voltage DC point after the DC fault. Without the auxiliary decision, the pre defined load control strategy is started without auxiliary decision, and the control instructions are sent to the fast response module of the marketing load. The load fast response module is used for the pre defined control users. Line control. After the UHV DC fault, the auxiliary decision control control the limit of the control section of the main station, the control of the overuse of the connection line, the control of the standby and so on. It automatically starts the auxiliary decision after the UHV fault, and calculates the load needed to be removed in real time. After the manual confirmation, the load control is carried out to the load quick response module.

Emergency coordination control technology of power grid .The emergency coordinated control of power network with friendly interaction of source network is an urgent interaction model of the power grid to respond to the lack of reserve capacity of the power grid and the limit of the power flow of the line or equipment. As the main way of emergency response, the main route of the power grid is to cut the interruptible load, low frequency load, low voltage load, active disassembly, DC power modulation and primary frequency modulation on-line monitoring, which can be adjusted and optimized when the power grid is insufficient or the power flow is limited. Through the proper control of the interruptible load, low frequency load reduction, low voltage load reduction, active disassembly and DC power modulation, and the optimization of the primary frequency modulation on-line monitoring system of the generator set, the transient stability of the system can be improved and the emergency coordinated control of the regional Source-grid-load and interaction can be realized.

Network load emergency coordination control technology. network charge friendly interaction control technology such as intelligent low frequency and low voltage load reduction measures, on the premise of ensuring the safety and stability of the power grid, on the one hand, we should consider all kinds of policies and regulations, energy saving and emission reduction and economic benefits and other constraints, on the other hand, the user's intention should be fully considered. Users are willing to participate in demand response control and reduce the compound control cost of load shedding.

Large scale power load group control technology. The scale power load group control, as a fast load reducing form, uses the virtual power plant resources with controllable regulation ability, and provides a centralized control type of real-time stable auxiliary service for the power grid.

The coordinated control technology of the charge second level / minute level of the source network. AGC emergency coordination control technology. After the UHV DC fault, the AGC one button urgently mobilize the unit to increase the output and implement the emergency switching of the unit control mode to support the rapid recovery of the frequency. Based on optimization adjustment auxiliary decision-making, preventive and corrective control is carried out for key sections to achieve safe closed loop control in power support and standby recovery process.

The load recovery technology of the source network charged second level / minute level. After the grid frequency is basically restored, under the condition of guaranteeing the security of the power grid and retaining the reserve requirements, the power generation capacity of the grid connected unit and the inter provincial connection line transport capacity are fully utilized, and the load recovery process of each area and zoning is decided and the power supply capacity is calculated. Considering the safety factors, the method of electricity quota is adopted to carry out orderly load recovery and reduce the power failure time of customers through local adjustment and regional negative control centers. 


\section{Conclusions}

In this paper, starting from the basic model of optimization control for coordination of source-grid-load, the interaction among source grid and load is summed up into six kinds; and combination with the theory, the demand environment of source-grid-load coordination optimization control is analyzed; finally, the corresponding key technologies are put forward. On the one hand, it helps to solve problems in time when the power grid is affected by UHV technology, so as to ensure the stable operation of the power grid. On the other hand, it provides theoretical and technical support for the stable operation of the power grid.

\section{References}

[1]Yang Li. Research on Optimization of power structure in China based on sustainable development [D]. Harbin Engineering University, 2010.

[2]Zhang Ning, Liu Jingkun. Factors affecting the operation of UHV power grids and Countermeasures [J]. power system protection and control, 2013,41 (01): 109-114.

[3] Zhao Liang, Guo Qiang, Qin Qin, Zhang Ke, Zhang Wenzhao, Tang Xiaojun, Zhang Yantao, Wang Qing. Analysis of stability characteristics of UHV synchronous grid [J]. Journal of electrical engineering of China, 2008 (34): 47-51.

[4]Luo Jianyu, Li Haifeng, Jiang Ye Feng, Luo Kaiming, Liu Lin. Based on stability control technology, Source-grid-load friendly interactive precision load control system [J]. power engineering technology, 2017,36 (01): 25-29.

[5]Li Ming, Bao Zonglei, Zhang Qingyun, Zhou Weihua. Large-scale source network charged friendly interactive system $[\mathrm{J} / \mathrm{OL}]$ rural electrification, 2018 (03): 39-41[2018-04-24].https://doi.org/10.13882/j.cnki.ncdqh.2018.03.013. 